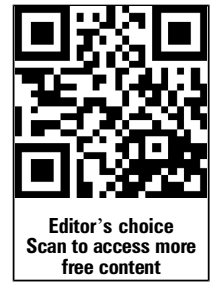

For numbered affiliations see end of article.

Correspondence to Dr A S Turk, Department of Radiology and Radiological Sciences, Medical University of South Carolina, 96 Jonathan Lucas Street, CSB 210, Charleston, SC 29425, USA; turk@musc.edu

Received 16 January 2014 Revised 4 February 2014 Accepted 5 February 2014 Published Online First 25 February 2014

\section{SLinked}

http://dx.doi.org/10.1136/ neurintsurg-2014-011187

CrossMark

To cite: Turk AS, Frei $D$, Fiorella D, et al. J Neurolntervent Surg 2014;6:260-264.

\title{
ADAPT FAST study: a direct aspiration first pass technique for acute stroke thrombectomy
}

Aquilla S Turk, ${ }^{1}$ Don Frei, ${ }^{2}$ David Fiorella, ${ }^{3}$ J Mocco, ${ }^{4}$ Blaise Baxter, ${ }^{5}$ Adnan Siddiqui, ${ }^{6}$ Alex Spiotta, ${ }^{7}$ Maxim Mokin, ${ }^{3}$ Michael Dewan, ${ }^{8}$ Steve Quarfordt, ${ }^{5}$ Holly Battenhouse, ${ }^{9}$ Raymond Turner, ${ }^{7}$ Imran Chaudry ${ }^{1}$

\begin{abstract}
Background The development of new revascularization devices has improved recanalization rates and time, but not clinical outcomes. We report a prospectively collected clinical experience with a new technique utilizing a direct aspiration first pass technique with large bore aspiration catheter as the primary method for vessel recanalization. Methods 98 prospectively identified acute ischemic stroke patients with 100 occluded large cerebral vessels at six institutions were included in the study. The ADAPT technique was utilized in all patients. Procedural and clinical data were captured for analysis.
\end{abstract}

Results The aspiration component of the ADAPT technique alone was successful in achieving Thrombolysis in Cerebral Infarction ( $\mathrm{TICI}$ ) 2b or 3 revascularization in $78 \%$ of cases. The additional use of stent retrievers improved the $\mathrm{TICl} 2 \mathrm{~b} / 3$ revascularization rate to $95 \%$. The average time from groin puncture to at least $\mathrm{TICl} 2 \mathrm{~b}$ recanalization was $37 \mathrm{~min}$. A $5 \mathrm{MAX}$ demonstrated similar success to a 5MAX ACE in achieving $\mathrm{TICl} 2 \mathrm{~b} / 3$ revascularization alone ( $75 \%$ vs $82 \%, p=0.43$ ). Patients presented with an admitting median National Institutes of Health Stroke Scale (NIHSS) score of 17.0 (12.0-21.0) and improved to a median NIHSS score at discharge of 7.3 (1.0-11.0). Ninety day functional outcomes were 40\% (modified Rankin Scale (mRS) 0-2) and 20\% (mRS 6). There were two procedural complications and no symptomatic intracerebral hemorrhages.

Discussion The ADAPT technique is a fast, safe, simple, and effective method that has facilitated our approach to acute ischemic stroke thrombectomy by utilizing the latest generation of large bore aspiration catheters to achieve previously unparalleled angiographic outcomes.

\section{INTRODUCTION}

Early and efficient revascularization of large vessel occlusions has been shown to correlate with improved outcomes in selected patients with acute ischemic stroke. ${ }^{1-3}$ Aspiration thrombectomy using the Penumbra system, while an effective technique for achieving revascularization, has yielded only modest clinical results. ${ }^{4-6}$ Stent retrievers have also been shown to be effective for vessel recanalization, with similar clinical outcomes. ${ }^{2} 3$

Recent advances in catheter technology have included very large, easily trackable, aspiration thrombectomy catheters that can now more easily and reliably navigate the cerebrovasculature. A novel technique using this newest generation of large bore aspiration catheters as a first approach for thrombectomy has recently been reported. ${ }^{7}$ The purpose of this study was to follow-up the initial experience with a prospective report of all stroke cases that had undergone a direct aspiration first pass technique (ADAPT) with a large bore aspiration catheter as the primary method for vessel recanalization.

\section{METHODS}

Under an institutional review board approved protocol, prospectively identified patient data were collected on all stroke patients undergoing the ADAPT technique at the Medical University of South Carolina, Swedish Medical Center, Vanderbilt University, Stonybrook University, Erlanger Medical Center, and University of Buffalo. Patients were selected for intervention as per the investigator protocol at each site. Usual practice was advanced imaging with CT/CT angiography and CT perfusion for patient selection. Patients included in this study were identified to have a large vessel cerebral vessel occlusion with viable ischemic penumbra and less than one-third ischemic vascular territory.

The ADAPT technique has been described previously. ${ }^{7}$ Briefly, a large guide catheter (Neuron 088; Penumbra, Oakland, California, USA) was advanced as far distally into the cervical or proximal petrous internal carotid artery (ICA) as possible. The largest caliber aspiration catheter that the vessel would accommodate was selected for each case, usually a 5MAX (Penumbra) or 5MAX ACE (Penumbra), for distal ICA, proximal middle cerebral artery, and basilar occlusions. This catheter was advanced to the level of the thrombus, usually coaxially over a 016 microwire (Fathom; Boston Scientific Corp, Fremont, California, USA) and Velocity microcatheter (Penumbra). Other obturating catheters, such as $3 \mathrm{MAX}$ or smaller microcatheters, can also be used in conjunction with any compatible microwire. With the large bore aspiration catheter at the face of the thrombus, aspiration was applied with either a 20 or $60 \mathrm{~mL}$ syringe or Penumbra aspiration pump. Absence of flow within the aspiration system confirmed engagement with the thrombus. At this point, the catheter was gently advanced for $1-2 \mathrm{~mm}$ to ensure solid engagement with the thrombus. Aspiration was left for approximately $20 \mathrm{~s}$, and if no flow through the system was found then the catheter 
was slowly withdrawn. If aspiration failed, then the large aspiration catheter was reinserted up to the level of the thrombus and repeat aspiration attempted. At the operator's discretion, additional devices (such as stent retrievers) were used if aspiration alone failed.

Patient demographic, angiographic, and clinical data were collected. The degree of vessel occlusion before and after treatment was defined by the modified Thrombolysis in Cerebral Infarction (TICI) classification and adjudicated by the operator. Successful revascularization was defined as a TICI score $\geq 2 b$ post-treatment. Procedure time was defined as the time from groin access to at least TICI $2 \mathrm{~b}$ revascularization. Symptomatic intracerebral hemorrhage (sICH) was defined as presence of hemorrhage after treatment, with worsening of clinical examination by $\geq 4$ points on the National Institutes of Health Stroke Scale (NIHSS). The 90 day functional outcomes were determined by the modified Rankin Scale (mRS), and were obtained from the available clinic records. A good functional outcome was defined as an mRS score of $\leq 2$ at 90 days. Mortality was defined as death occurring within 90 days of initial presentation. The 5MAX ACE became clinically available during the present study. Outcomes for 5MAX and 5MAX ACE were compared.

Data were tracked at each site. De-identified data were submitted to the sponsoring institution (Medical University of South Carolina) which performed the primary analysis. Statistical analyses were performed using SAS V.9.3 (SAS Institute, Cary, North Carolina, USA). Differences between groups were tested using Student's $t$ test for continuous measures and a $\chi^{2}$ test for categorical measures. Differences between groups were tested using Fisher's exact test for categorical measures with expected cell sizes $<5$. All tests were two sided and assessed at a significance level of 0.05 .

\section{RESULTS}

\section{Patient demographics and procedural data}

Ninety-eight patients with 100 occlusions were treated at the six institutions, including 46 women, with an average age of 66 years (median 69, SD=15.7). The average time from when the patient was last seen normal to groin puncture was $8.5 \mathrm{~h}$ (mean $507 \mathrm{~min}$; median $241.5 \mathrm{~min}, \mathrm{SD}=506 \mathrm{~min}$ ). The overall successful revascularization rate (TICI 2b-3) was 95\%. The average time to TICI $2 \mathrm{~b}$ or 3 revascularization was $36.6 \mathrm{~min}$ $(\mathrm{SD}=26.4 \mathrm{~min})$. The aspiration component of the ADAPT technique alone was successful in achieving successful revascularization of the occluded vessel $78 \%$ of the time. When the aspiration component of ADAPT was successful as a standalone technique, the average time from femoral access to final revascularization was $31.6 \mathrm{~min}(\mathrm{SD}=23.3 \mathrm{~min})$. In those cases where an adjunctive device was required, revascularization times were significantly $\quad(\mathrm{p}<0.0001) \quad$ prolonged (average $56.8 \mathrm{~min}$ $(\mathrm{SD}=29.1 \mathrm{~min}))$.

Ten of $100(10 \%)$ cases had downstream emboli within the initially affected territory, all of which were subsequently removed with either subsequent aspiration at the occlusion site or utilization of a stent retriever. There were no instances of embolization to a new territory (ENT). Two (2\%) device related complications were encountered. One case was related to vessel dissection trying to advance the 5MAX catheter through an occlusion that after aspiration thrombectomy revealed an underlying intracranial stenosis, which was successfully stented. One complication was related to advancing a 5MAC ACE from the distal ICA into the mid M1 middle cerebral artery without using an obturating microwire or microcatheter, and resulted in significant vessel dissection, which was unable to be opened.
Table 1 Comparison of the 5MAX and 5MAX ACE final outcomes

\begin{tabular}{llll}
\hline & 5MAX & 5MAX ACE & p Value \\
\hline No of cases & 44 & 44 & \\
Mean time to revascularization (min) & 37.7 & 35.6 & 0.71 \\
TICI 2b (\%) & 54.6 & 36.4 & 0.09 \\
TICl 3 (\%) & 40.9 & 61.4 & 0.06 \\
mRS 0-2 (\%) & 34 & 50 & 0.19 \\
\hline
\end{tabular}

TICI 3 revascularization was achieved more frequently with $5 \mathrm{MAX}$ ACE than with 5MAX (61.4\% vs $40.9 \% ; p=0.055)$.

mRS, modified Rankin Scale; TICl, Thrombolysis in Cerebral Infarction.

The primary ADAPT revascularization catheter was 5MAX in 44 cases, 5MAX ACE in 44 cases, 4MAX (Penumbra) in six cases, 3MAX (Penumbra) in four cases, Navien 058 (EV3 Covidien) in one case, and Neuron 088 MAX (Penumbra) in one case. TICI $2 \mathrm{~b}$ or 3 revascularization with aspiration alone was achieved in $75 \%$ of cases in which a 5 MAX was the primary catheter versus $82 \%(\mathrm{p}=0.44)$ when a $5 \mathrm{MAX}$ ACE was used (table 1).

\section{Clinical outcomes}

There were no incidences of postprocedure sICH. The presenting NIHSS was 17.2 (median 17.0; $\mathrm{SD}=6.4$ ) on average and improved to an average of 7.3 (median $4.0 ; \mathrm{SD}=7.5$ ) at discharge (table 2).

mRS was available in 81 of 98 patients (83\%) (table 3). Forty per cent of patients achieved an mRS score of 0-2 and $20 \%$ an mRS score of 6 at the 3 month follow-up. In the $78 \%$ of cases

Table 2 Baseline characteristics

\begin{tabular}{|c|c|}
\hline Variable & \\
\hline Mean age (years) & 66.3 \\
\hline \multicolumn{2}{|l|}{ Gender } \\
\hline Men (n (\%)) & $46(47)$ \\
\hline Women (n (\%)) & $52(53)$ \\
\hline \multicolumn{2}{|l|}{ NIHSS } \\
\hline Pretreatment & $17.2 / 17.0^{*}$ \\
\hline Post-treatment & $7.3 / 4.0^{*}$ \\
\hline \multicolumn{2}{|l|}{ IV tPA } \\
\hline Yes (n (\%)) & $27(28)$ \\
\hline No (n (\%)) & $70(72)$ \\
\hline Average time to groin puncture (h) & 8.5 \\
\hline Average time to $\mathrm{TICl} 2 \mathrm{~b} / 3$ recanalization (min) & 37 \\
\hline \multicolumn{2}{|l|}{ Site of occlusion (n (\%)) } \\
\hline Right M1 & $20(20)$ \\
\hline Right M2 & $11(11)$ \\
\hline Right ICA & $3(3)$ \\
\hline Right ICA terminus & $3(3)$ \\
\hline Left M1 & $23(23)$ \\
\hline Left M2 & $7(7)$ \\
\hline Left ICA & $6(6)$ \\
\hline Left ICA terminus & $11(11)$ \\
\hline Basilar & $5(5)$ \\
\hline Right cervical ICA-MCA & $8(8)$ \\
\hline Left cervical ICA-MCA & $3(3)$ \\
\hline
\end{tabular}


Table 3 Demographics and outcomes of ADAPT FAST cases

\begin{tabular}{ll}
\hline Outcome parameter & \\
\hline TICl pretreatment (n (\%)) & \\
0 & $96(96)$ \\
1 & $1(1)$ \\
$2 \mathrm{a}$ & $3(3)$ \\
TICl post-treatment (n (\%)) & \\
$0-2 \mathrm{a}$ & $5(5)$ \\
$2 \mathrm{~b}$ & $44(44)$ \\
3 & $51(51)$ \\
NIHSS & \\
Pretreatment & 17.2 \\
Post-treatment & 7.3 \\
90 day mRS (n (\%)) & \\
0 & $10(13)$ \\
1 & $16(20)$ \\
2 & $5(6)$ \\
3 & $12(15)$ \\
4 & $11(14)$ \\
5 & $8(10)$ \\
6 & $15(19)$ \\
Missing & $23(23)$ \\
sICH (n (\%)) & $0(0)$ \\
\hline The90 day mRS outcomes calculated on percentage of patients that had a follow-up \\
$(\mathrm{n}=79)$. & \\
mRS, modified Rankin Scale; NIHSS, National Institutes of Health Stroke Scale; sICH, \\
symptomatic intracerebral hemorrhage; TICl, Thrombolysis in Cerebral Infarction.
\end{tabular}

where ADAPT alone was successful, $47 \%$ of patients achieved an mRS score of $0-2$ and $14 \%$ resulted in an mRS score of 6 . In the cases where ADAPT required a stent retriever or was unsuccessful, 18\% achieved mRS 0-2 and 35\% resulted in mRS 6.

\section{DISCUSSION}

Catheter aspiration thrombectomy has been reported previously, but as either a bailout after traditional techniques had failed or as a strategy by which to achieve revascularization of an occluded large extracranial artery. ${ }^{8-12}$ ADAPT represents a novel revascularization strategy made possible by the availability of newer, flexible, atraumatic, large bore aspiration catheters.
These catheters are easily navigated into the intracranial circulation and provide a large cross sectional area for the aspiration and engagement of the occluding thrombus. This technique is based on using aspiration alone as the primary mechanism of thrombectomy and, if initially unsuccessful, then incorporating adjunctive alternatives such as a stent retriever to achieve revascularization. The technique was successful alone in achieving TICI $2 \mathrm{~b} / 3$ revascularization in $78 \%$ of cases or with adjunctive devices in $95 \%$ of cases. On average, revascularization was achieved within $37 \mathrm{~min}$ of groin puncture, and in $15 \mathrm{~min}$ or less from groin puncture in more than 20 cases. These efficient revascularization metrics were manifest clinically, with average discharge NIHSS improvement of 10 points and with favorable clinical functional outcomes (mRS $0-2$ at 90 days) in $40 \%$ of cases.

Compared with the outcomes of stent retriever data, ADAPT yielded similar rates of good functional outcome (mRS 0-2), mortality, and device related complications (table 4). ${ }^{2} 3$ 13-16

In the present series using ADAPT, fewer sICH were observed, and times to recanalization were, on average, probably shorter. It is likely that ADAPT produced higher rates of recanalization; however, this is difficult to establish with the documented methods of assessing successful recanalization used in the predicate stent retriever studies. With ADAPT, TICI $2 \mathrm{~b}-3$ revascularization was achieved with aspiration alone in $78 \%$ of cases compared with rates of TICI $2 \mathrm{a}-3$ recanalization (ranging from $68 \%$ to $83 \%$ ) for stent retrievers. A modified TICI score of $2 \mathrm{a}$ indicates a large area of non-perfused brain parenchyma and is increasingly not considered technically successful revascularization. ${ }^{17}$ The final procedural TICI $2 \mathrm{~b} / 3$ revascularization was 95\% with ADAPT, which is higher than that achieved in the STAR study (85\%) and in the NASA study (76\%). ${ }^{14} 16$

The NASA registry recently reported an organized retrospective experience of 354 stroke cases from 24 centers. ${ }^{14}$ The overall results were similar to those reported in the meta-analysis by Walcott et al. ${ }^{13}$ A subgroup analysis from the NASA study was subsequently reported to determine if the use of a balloon guide catheter (BGC) improved outcomes. ${ }^{18}$ The use of a BGC significantly improved TICI 3 revascularization rates to $54 \%$, although overall TICI $2 \mathrm{~b} / 3$ revascularization rates remained similar at $75 \%$. This is similar to the TICI 3 rate of $51 \%$ but remains lower than the final $95 \%$ TICI $2 \mathrm{~b} / 3$ revascularization we were able to achieve with ADAPT. Stent retriever

Table 4 Comparison of outcomes from current ADAPT FAST, SWIFT, TREVO, NASA, and stent retriever meta-analysis

\begin{tabular}{|c|c|c|c|c|c|c|c|}
\hline & $\begin{array}{l}\text { ADAPT FAST } \\
(n=100)\end{array}$ & $\begin{array}{l}\text { SWIFT trial } \\
(n=89)\end{array}$ & $\begin{array}{l}\text { TREVO trial } \\
(n=88)\end{array}$ & $\begin{array}{l}\text { STAR study } \\
(n=202)\end{array}$ & $\begin{array}{l}\text { NASA registry } \\
(n=354)\end{array}$ & $\begin{array}{l}\text { Solitaire data* } \\
(n=355)\end{array}$ & $\begin{array}{l}\text { Trevo data* } \\
(n=221)\end{array}$ \\
\hline $\mathrm{TICl} 2 \mathrm{a} / 2 \mathrm{~b} / 3(\%)$ & $\mathrm{N} / \mathrm{A}$ & $83+$ & 90 & N/A & 87.5 & 82 & 83 \\
\hline Device $\mathrm{TICl} 2 \mathrm{~b} / 3(\%)$ & 78 & $75.9 \ddagger$ & $68 \ddagger$ & $\begin{array}{l}79 \ddagger \\
91 \S\end{array}$ & 72.5 & NR & NR \\
\hline Final $\mathrm{TICl} 2 \mathrm{~b} / 3(\%)$ & 95 & $75.9 \ddagger$ & $68 \ddagger$ & 85 & 72.5 & 82 & 83 \\
\hline mRS 0-2 (\%) & 40 & 36 & 40 & 55 & 42 & 47 & 51 \\
\hline Mortality (\%) & 20 & 17 & 34 & 19 & 30.2 & 14 & 31 \\
\hline Time to final revascularization (min) & 37 & NR & NR & NR & 509 & NR & NR \\
\hline Device related complications (\%) & 2 & 8.6 & 9 & 7.5 & NR & 6 & 5 \\
\hline Symptomatic ICH (\%) & 0 & 2 & 7 & 4 & 9.9 & 6 & 8 \\
\hline
\end{tabular}

${ }^{*}$ Meta-analysis of real world published experience.

† Site reported rate of recanalization.

$\neq$ Core laboratory reported.

$\S$ Site investigator reported data.

१Time to revascularize after guide catheter access.

ICH, intracerebral hemorrhage; mRS, modified Rankin Scale; N/A, not applicable; NR, not reported; TICl, Thrombolysis in Cerebral Infarction. 
procedures have been shown to be significantly faster with use of a BGC (average of $120 \mathrm{~min}$ ), which is markedly longer than the average 38 min ADAPT case. ${ }^{16}$ However, the times are difficult to compare as the BGC definition of procedure time was from groin access to groin closure whereas the ADAPT procedure time was groin access to at least TICI $2 b$ revascularization. Finally, average discharge NIHSS score in those with BGC was 12 versus 17.5 when a BGC was not used, which is higher than our average discharge NIHSS score of 7.

One main advantage of ADAPT as a firstline approach is that it is versatile. The technique does not preclude the operator from incorporating other devices if aspiration alone is not working. Having the large bore aspiration catheter at the face of the clot facilitates the use of adjunctive devices, such as stent retrievers, as it provides a direct conduit to the thrombus. The technique of using stent retrievers in conjunction with local aspiration at the face of the clot has also been reported, with very high rates of technical success and good neurological outcomes. This approach was originally proposed to address the high incidence of ENT that has been reported with thrombectomy. ${ }^{19}$ Kurre et $a l^{19}$ reported in 175 occluded vessels a TICI $2 \mathrm{~b} / 3$ revascularization rate of $91 \%$ and an improvement in ENT to $3.5 \%$ from $14 \%$ when distal aspiration was used with stent retrievers. Using combined local aspiration with stent retrieval, ${ }^{20}$ Humphries et $a l^{20}$ recently reported minimal ENT $(<5 \%)$ with very high rates of revascularization (TICI $2 \mathrm{~b}-3$ of $88 \%$ ) and low $(5 \%)$ sICH rates. This approach is also being validated in the ongoing Penumbra three-dimensional separator trial.

ADAPT is technically straightforward, requiring only the navigation of a catheter to the face of the occlusion followed by the application of aspiration. The operator typically does not have to completely traverse the occlusion, and in most cases no additional devices have to be deployed or manipulated. Aspiration alone with the larger 5MAX ACE (0.060 inner diameter) catheter resulted, on average, in $82 \%$ TICI $2 \mathrm{~b} / 3$ revascularization rates compared with the original $5 \mathrm{MAX}(0.054$ inner diameter) catheter of $75 \%$. This validates the intuitive concept of the improved aspiration performance of a larger bore catheter. In addition, clot extraction with ADAPT places little or no traction on the parent artery and regional penetrating arteries. As such, the potential for endothelial injury is likely to be far lower, possibly accounting for the absence of sICH in our current series. ${ }^{21}$ The ability of ADAPT to engage the face of the thrombus and avoid superselective contrast injections into the ischemic region may also contribute to the lower hemorrhage rate. ${ }^{22}$ Intracranial hemorrhage reportedly occurs in as many as $41 \%$ of stroke patients after thrombectomy with stent retrievers, of which approximately one-fifth lead to a symptomatic deterioration. $^{2} 351418$

ADAPT was, on average, faster than traditional methods of thrombectomy, particularly when the strategy worked as a standalone technique, as it did in the majority of cases. Moreover, thrombus which was difficult to extract with ADAPT, and was also typically challenging to clear with adjunctive devices, reflected in the marked prolongation of procedural times (57 vs $32 \mathrm{~min}$ ) observed in those cases in which adjunctive devices were required.

ADAPT seemed to cause minimal clot disruption and fragmentation, often allowing the extraction of the occlusive embolus en bloc with a single pass. This may decrease the frequency and number of downstream emboli. In vitro tests have shown that stent retriever and traditional aspiration thrombectomy (performed with a separator) result in fragmentation of the embolus during extraction, creating downstream emboli and potentially emboli to previously uninvolved vascular territories. $^{23}$ When fragmentation of the embolus did occur with ADAPT (observed in $10 \%$ of cases in the present series), the emboli were usually large enough and proximal enough to allow extraction using aspiration alone with a smaller catheter, such as 3 MAX in the M2 middle cerebral artery segment or a stent retriever.

Similar functional outcomes (mRS 0-2) of 40\% were found with ADAPT as with other previously reported thrombectomy strategies, ${ }^{2} 351418$ despite a relatively long average time $(8.5 \mathrm{~h})$ from symptom onset to treatment in the present series. These rates of favorable neurological outcome are similar to those reported in previous studies using a modern perfusion guided imaging paradigm to select patients for intervention rather than time alone. ${ }^{24}$ The variability in patient selection can affect clinical outcomes and is a limitation of this study. It is important to consider however, that the operator reported assessments in the current study are more prone to bias in comparison with independently assessed and monitored outcomes reported in some of the comparative thrombectomy device trials.

\section{CONCLUSION}

This multicenter series supports the hypothesis that, in comparison with modern thrombectomy techniques, ADAPT is a fast, simple, efficient, and safe strategy to achieve revascularization in patients with acute ischemic stroke secondary to a large vessel occlusion. In the minority of cases in which aspiration alone is unsuccessful in achieving complete revascularization, the platform is versatile, allowing the rapid incorporation of adjunctive devices (such as stent retrievers).

\section{Author affiliations}

${ }^{1}$ Department of Radiology, Medical University of South Carolina, Charleston, South Carolina, USA

${ }^{2}$ Department of Interventional Neuroradiology, Radiology Imaging Associates, Englewood, Colorado, USA

${ }^{3}$ Department of Neurological Surgery, Stony Brook Medicine, Stony Brook, New York, USA

${ }^{4}$ Department of Radiology and Radiological Sciences, Vanderbilt University, Nashville, Tennessee, USA

${ }^{5}$ Tennessee Interventional Associates, Chattanooga, Tennessee, USA

${ }^{6}$ Department of Neurosurgery, University of Buffalo, Buffalo, New York, USA

${ }^{7}$ Division of Neurosciences, Department of Neurosurgery, Medical University of South Carolina, Charleston, South Carolina, USA

${ }^{8}$ Department of Neurological Surgery, Vanderbilt University, Nashville, Tennessee, USA

${ }^{9}$ Department of Public Health Sciences, Medical University of South Carolina, Charleston, South Carolina, USA

Contributors Each author listed above should receive authorship credit based on material contribution to the article, revision of the article, and final approval of the article for submission to this journal.

Competing interests AST reports grants and non-financial support from Penumbra, grants from Microvention, grants and non-financial support from Stryker, non-financial support from Microvention, non-financial support from Covidien, non-financial support from Siemens, and grants and non-financial support from Boston Scientific, outside the submitted work. BB reports non-financial support from Stryker, non-financial support from Penumbra, non-financial support from Covidien, non-financial support from Silk Road, non-financial support from Reverse Medical, and non-financial support from Rapid Medical, outside the submitted work. AS reports non-financial support from Pulsar Vascular and grants from Simbionix, outside the submitted work. JM reports non-financial support from Lazarus Effect, non-financial support from Medina Medical, non-financial support from Pulsar Vascular, non-financial support from Reverse Medical, and non-financial support from Edge Therapeutics, outside the submitted work. RT reports grants and non-financial support from Penumbra, grants and non-financial support from Microvention, and grants and non-financial support from Stryker, outside the submitted work. IC reports grants and non-financial support from Penumbra, grants and non-financial support from Microvention, and non-financial support from Stryker, outside the submitted work. 
Ethics approval The study was approved by the institutional review board of the Medical University of South Carolina.

Provenance and peer review Not commissioned; externally peer reviewed.

\section{REFERENCES}

1 Rha JH, Saver JL. The impact of recanalization on ischemic stroke outcome: a meta-analysis. Stroke 2007;38:967-73.

2 Nogueira RG, Lutsep HL, Gupta R, et al. Trevo versus Merci retrievers for thrombectomy revascularisation of large vessel occlusions in acute ischaemic stroke (TREVO 2): a randomised trial. Lancet 2012;380:1231-40.

3 Saver JL, Jahan R, Levy El, et al. Solitaire flow restoration device versus the Merci retriever in patients with acute ischaemic stroke (SWIFT): a randomised, parallel-group, non-inferiority trial. Lancet 2012;380:1241-9.

4 Frei $D$, Gerber J, Turk $A$, et al. The SPEED study: initial clinical evaluation of the Penumbra novel 054 reperfusion catheter. J Neurointerv Surg 2013;5(Suppl 1):i74-6.

5 Tarr R, Hsu D, Kulcsar Z, et al. The POST trial: initial post-market experience of the Penumbra system: revascularization of large vessel occlusion in acute ischemic stroke in the United States and Europe. J Neurointerv Surg 2010;2:341-4.

6 Turk AS III, Campbell JM, Spiotta A, et al. An investigation of the cost and benefit of mechanical thrombectomy for endovascular treatment of acute ischemic stroke. J Neurointerv Surg 2014;6:77-80.

7 Turk AS, Spiotta A, Frei D, et al. Initial clinical experience with the ADAPT technique: a direct aspiration first pass technique for stroke thrombectomy. J Neurointerv Surg 2014;6:231-7.

8 Iwata T, Mori T, Tajiri H. Successful removal of clots from embolic carotid occlusion by aspiration with a syringe-case report. Brain Nerve 2008;60:1067-72.

9 Jankowitz B, Aghaebrahim A, Zirra A, et al. Manual aspiration thrombectomy: adjunctive endovascular recanalization technique in acute stroke interventions. Stroke 2012;43:1408-11.

10 Jankowitz BT, Aleu A, Lin R, et al. Endovascular treatment of basilar artery occlusion by manual aspiration thrombectomy. J Neurointerv Surg 2010;2:110-14.

11 Spiotta AM, Hussain MS, Sivapatham T, et al. The versatile distal access catheter: the Cleveland Clinic experience. Neurosurgery 2011;68:1677-86.

12 Xu GF, Suh DC, Choi CG, et al. Aspiration thrombectomy of acute complete carotid bulb occlusion. J Vasc Interv Radiol 2005;16:539-42.
13 Walcott BP, Boehm KM, Stapleton CJ, et al. Retrievable stent thrombectomy in the treatment of acute ischemic stroke: analysis of a revolutionizing treatment technique. J Clin Neurosci 2013:20:1346-9.

14 Zaidat 00, Castonguay AC, Gupta R, et al. North American Solitaire Stent Retriever Acute Stroke registry: post-marketing revascularization and clinical outcome results. J Neurointerv Surg Published Online First: 23 Sep 2013. doi:10.1136/ neurintsurg-2013-010895

15 Davalos A, Pereira VM, Chapot R, et al. Retrospective multicenter study of Solitaire FR for revascularization in the treatment of acute ischemic stroke. Stroke 2012:43:2699-705.

16 Pereira VM, Gralla J, Davalos A, et al. Prospective, multicenter, single-arm study of mechanical thrombectomy using Solitaire Flow Restoration in acute ischemic stroke. Stroke 2013;44:2802-7.

17 Fargen $\mathrm{KM}$, Meyers $\mathrm{PM}$, Khatri $\mathrm{P}$, et al. Improvements in recanalization with modern stroke therapy: a review of prospective ischemic stroke trials during the last two decades. J Neurointerv Surg 2013:5:506-11.

18 Nguyen TN, Malisch T, Castonguay AC, et al. Balloon guide catheter improves revascularization and clinical outcomes with the Solitaire device: analysis of the North American Solitaire Acute Stroke Registry. Stroke 2014;45:141-5.

19 Kurre W, Aguilar-Perez M, Fischer $\mathrm{H}$, et al. How to prevent ACA emboli during mechanical thrombectomy of M1 occlusions. Presented at the ABC-WIN Seminar. Val d'Isere, France: ABC-WIN, 2013

20 Humphries W, Hoit D, Doss VT, et al. Distal aspiratin with retrievable stent assisted thrombectomy for the treatment of acute ischemic stroke. J Neurolntervent Surg Published Online First: 24 Jan 2014. doi:10.1136/neurintsurg-2013-010986

21 Khalessi A. Patterns of endothelial trauma in mechanical thrombectomy for acture ischemic stroke: Lessons from a live cell artificial vessel system for Penumbra, TREVO and Solaitaire FR. ABC-WIN; Val d'Isere, France, 2014.

22 Khatri R, Khatri $P$, Khoury J, et al. Microcatheter contrast injections during intra-arterial thrombolysis increase intracranial hemorrhage risk. J Neurointerv Surg 2010;2:115-19.

23 Chueh JY, Wakhloo AK, Gounis MJ. Effectiveness of mechanical endovascula thrombectomy in a model system of cerebrovascular occlusion. AJNR Am J Neuroradiol 2012;33:1998-2003.

24 Turk AS, Magarick JA, Frei D, et al. CT perfusion-guided patient selection for endovascular recanalization in acute ischemic stroke: a multicenter study. J Neurointerv Surg 2013;5:523-7. 